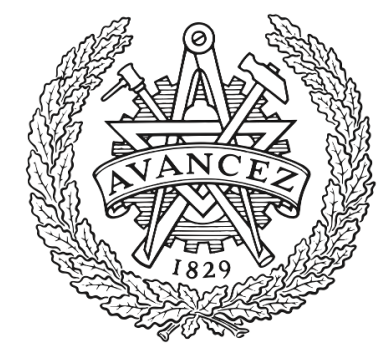

CHALMERS

UNIVERSITY OF TECHNOLOGY

\title{
Nonequilibrium effects in tunnel Josephson junctions
}

Downloaded from: https://research.chalmers.se, 2023-04-26 14:32 UTC

Citation for the original published paper (version of record):

Bezuglyi, E., Vasenko, A., Shumeiko, V. et al (2005). Nonequilibrium effects in tunnel Josephson junctions. Physical Review B - Condensed Matter and Materials Physics, 72. http://dx.doi.org/10.1103/PhysRevB.72.014501

N.B. When citing this work, cite the original published paper. 


\title{
Nonequilibrium effects in tunnel Josephson junctions
}

\author{
E. V. Bezuglyi, ${ }^{1,2}$ A. S. Vasenko, ${ }^{2,3}$ V. S. Shumeiko, ${ }^{2}$ and G. Wendin ${ }^{2}$ \\ ${ }^{1}$ Institute for Low Temperature Physics and Engineering, Kharkov 61103, Ukraine \\ ${ }^{2}$ Chalmers University of Technology, S-41296 Göteborg, Sweden \\ ${ }^{3}$ Department of Physics, Moscow State University, Moscow 119992, Russia
}

(Received 24 October 2004; revised manuscript received 15 February 2005; published 1 July 2005)

\begin{abstract}
We study nonequilibrium effects in current transport through voltage biased tunnel junction with long diffusive superconducting leads at low applied voltage $\mathrm{eV} \ll 2 \Delta$, and finite temperatures. Due to a small value of the Josephson frequency, the quasiparticle spectrum adiabatically follows the time evolution of the superconducting phase difference, which results in the formation of oscillating bound states in the vicinity of the tunnel junction (Andreev band). The quasiparticles trapped by the Andreev band generate higher even harmonics of the Josephson ac current, and also, in the presence of inelastic scattering, a nonequilibrium dc current, which may considerably exceed the dc quasiparticle current given by the tunnel model. The distribution of traveling quasiparticles also deviates from the equilibrium due to the spectrum oscillations, which results in an additional contribution to the de current, proportional to $\sqrt{V}$.
\end{abstract}

DOI: 10.1103/PhysRevB.72.014501

PACS number(s): 74.45.+c, 74.40.+k, 74.25.Fy, 74.50.+r

\section{INTRODUCTION}

Tunnel current transport in superconducting junctions is a classical topic of interest and research. ${ }^{1}$ Theoretical description of the superconducting tunneling given by the tunnel model $^{2,3}$ is based on the assumption about equilibrium in the junction electrodes. The resulting equations for the tunnel current are expressed through the nonperturbed density of states (DOS) and equilibrium distribution function. Such an approach includes only single-particle tunneling processes, and it is sufficient for describing current-voltage characteristics at large applied voltage $\mathrm{eV}>2 \Delta$, and within the subgap voltage region $\mathrm{eV}<2 \Delta$ at nonzero temperature. At very small temperatures, multiparticle tunneling processes must be taken into account. ${ }^{4,5}$ However, these processes are exponentially weak at small voltage, and if the temperature is not particularly small $\mathrm{eV} \ll T<2 \Delta$, only tunneling of thermally excited quasiparticles plays a significant role. It is clear, however, that quasiparticle tunneling generates nonequilibrium distribution in the electrodes, and that the effect is enhanced in diffusive junctions because of the scattering by impurities. Traditionally, this effect is considered to be negligibly small since it is of a higher order in the junction transparency.

How small is the nonequilibrium effect actually? In voltage biased superconducting junctions, the phase of the order parameter has different time dependencies in different electrodes, and the interference of the order parameters induced by the tunneling leads to the time oscillations of the DOS. The character of these oscillations can be qualitatively understood from comparison with the well-studied case of ballistic tunnel junctions. ${ }^{6-8}$ In the latter, the Andreev levels are formed in the vicinity of the tunnel barrier; their energies lie within the energy gap in the bulk electrodes, and oscillate in time following the evolution of the superconducting phase difference. Similarly, one may expect that in diffusive junctions the time oscillation of the DOS will have the form of a "breathing" potential well localized in the vicinity of the tunnel barrier ("Andreev band"), which periodically, with the
Josephson period, traps and releases quasiparticles. Thus the quasiparticles spend a larger time within the junction area compared to the traveling time, which should generate strongly nonequilibrium quasiparticle distribution and, as a result, an appreciable change of the tunneling current. The effect should be most pronounced for a planar junction geometry sketched in Figs. 1(a) and 1(b), when the tunnel junction is connected to bulk electrodes via diffusive superconducting wires whose length exceeds the size of the Andreev band. Otherwise, as in diffusive point contacts, the proximity of large equilibrium reservoirs will suppress the DOS oscillations and hence the formation of the Andreev band because of rapid spreading out of the current.

In this paper we demonstrate that the nonequilibrium quasiparticle distribution generated by the nonstationary process of formation of the Andreev band during the tunneling considerably modifies the dc tunnel current at a small applied voltage. We show that the nonequilibrium effect results in a time dependent amplitude of all the three current components given by the tunnel model $^{2,3}$

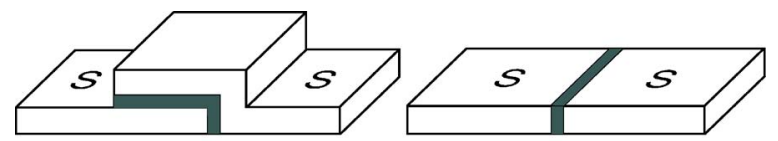

a)

b)

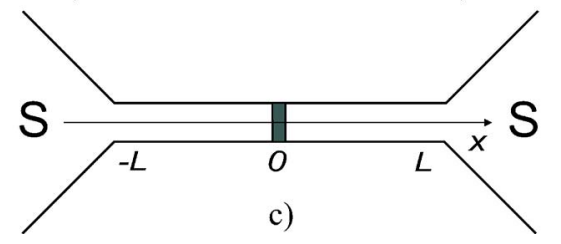

FIG. 1. (Color online) Possible experimental realizations of tunnel junctions (a), (b), and the theoretical model (c): The tunnel barrier attached to bulk superconducting electrodes by long ( $L$ $\gg \xi_{0}$ ) diffusive superconducting leads. 


$$
I(t)=I_{1}(t) \sin \phi+I_{2}(t)+I_{3}(t) \cos \phi, \quad \phi=2 \mathrm{eV} t
$$

(Josephson current, quasiparticle current, and the interference current, respectively). All the amplitudes have nonharmonic time dependence, and generally all of them contribute to the dc current. However, at a small voltage, the major contribution, nonanalytic in junction transparency and voltage, comes from the sine term (Josephson current). More precisely, this additional dc current results from a nonadiabatic component of the distribution function associated either with inelastic relaxation of quasiparticles, or with the quasiparticle diffusion away from the tunnel barrier. The additional dc current may considerably exceed the magnitude given by the tunnel model, and moreover, leads to a nonmonotonous net de current.

Such an effect has a close qualitative similarity to the nonequilibrium effects in transparent weak links [superconductor-normal metal-superconductor (SNS) junctions and superconducting bridges] studied earlier., 910 There are however the differences: In superconducting bridges, the potential well appears due to the suppression of the order parameter by the current concentration (depairing effect), ${ }^{9}$ while in the tunnel junctions this effect does not play any essential role. In long SNS junctions, the potential well is pre-prepared by the proximity effect, and it exists in the absence of the transport current; as a result, the oscillations develop at small energy, ${ }^{10}$ while in the tunnel junctions the oscillations occur at large energies close to the gap edge in the electrodes.

Organization of the paper is the following. In Sec. II we formulate the theoretical model and microscopic equations describing current transport through the tunnel junction with diffusive leads. In Sec. III we introduce an adiabatic approach for solving the time-dependent problem in the limit of low applied voltage. This approach is applied to the calculation of the spectral characteristics of the junction (Sec. IV) and the quasiparticle distribution function (Sec. V). In Sec. VI we calculate the ac and dc components of the net Josephson current, and then discuss and summarize the results in Secs. VII and VIII.

\section{MODEL AND BASIC EQUATIONS}

The model of the tunnel junction we are going to study is depicted in Fig. 1(c) and consists of a tunnel barrier with the transparency $\Gamma$ attached to bulk superconducting electrodes via two superconducting leads $(-L<x<0$ and $0<x<L)$. We will consider the diffusive limit, in which the elastic scattering length $\ell$ is much smaller than the coherence length $\xi_{0}=\sqrt{\mathcal{D} / 2 \Delta}$, where $\mathcal{D}$ is the diffusion coefficient (we assume $\hbar=k_{B}=1$ ). The length $L$ of the leads is assumed to be much larger than $\xi_{0}$ (long junction), and their width will be supposed to be much smaller than the Josephson penetration depth which implies homogeneity of the current along the junction. A similar model was considered in Refs. 11 and 12 in the study of the dc Josephson effect in tunnel structures.

Under these conditions, the microscopic calculation of the electric current $I(t)$ requires solution of the one-dimensional diffusive equations of nonequilibrium superconductivity ${ }^{13}$ (see also a review ${ }^{14}$ ) for the $4 \times 4$ matrix two-time KeldyshGreen's function $\breve{G}\left(x, t_{1}, t_{2}\right)$ in the leads,

$$
\begin{gathered}
{[\check{H}, \check{G}]=i \mathcal{D} \partial_{x} \check{J}, \quad \check{J}=\check{G} \circ \partial_{x} \check{G}, \quad \check{G}^{2}=\delta\left(t_{1}-t_{2}\right),} \\
\check{H}=\left[i \sigma_{z} \partial_{t_{1}}-e \varphi+\hat{\Delta}\left(t_{1}\right)\right] \delta\left(t_{1}-t_{2}\right),
\end{gathered}
$$

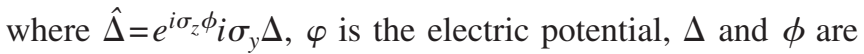
the modulus and the phase of the order parameter, respectively, $\sigma_{i}$ are the Pauli matrices, and

$$
\check{G}=\left(\begin{array}{cc}
\hat{g}^{R} & \hat{G}^{K} \\
0 & \hat{g}^{A}
\end{array}\right), \quad \hat{G}^{K}=\hat{g}^{R} \circ \hat{f}-\hat{f} \circ \hat{g}^{A} .
$$

In Eq. (3), $\hat{g}^{R, A}$ are the $2 \times 2$ Nambu matrix retarded and advanced Green's functions, and $\hat{f}=f+\sigma_{z} f_{-}$is the matrix distribution function (we use "check" for $4 \times 4$ and "hat" for $2 \times 2$ matrices). The multiplication procedure in Eqs. (2) and (3) is defined as the time convolution

$$
(A \circ B)\left(t_{1}, t_{2}\right)=\int_{-\infty}^{+\infty} A\left(t_{1}, t\right) B\left(t, t_{2}\right) d t .
$$

In Eqs. (2), we neglect the inelastic collision term, which will be taken into account later, in Sec. VI.

The boundary conditions for the function $\check{G}$ and the matrix current $\check{J}$ at the left $(x=-0)$ and the right $(x=+0)$ sides of the tunnel junction are given by relation ${ }^{15}$

$$
\check{J}_{-0}=\check{J}_{+0}=\left(2 g_{N} R\right)^{-1}\left[\check{G}_{-0}, \check{G}_{+0}\right],
$$

where $R$ is the junction resistance and $g_{N}$ is the normal conductivity of the leads per unit length. The electric current is related to the Keldysh component of the matrix current $\check{J}$ as

$$
I(t)=\left(\pi g_{N} / 4 e\right) \operatorname{Tr} \sigma_{z} \hat{J}^{K}(x, t, t)
$$

and thus it can be expressed through the boundary value $\check{J}_{0}$

$$
I(t)=\frac{\pi}{8 e R} \operatorname{Tr} \sigma_{z}\left[\check{G}_{-0}, \check{G}_{+0}\right]^{K}(t, t) .
$$

Equations (2) can be decomposed into the diffusion equations for the Green's functions

$$
[\hat{H}, \hat{g}]=i \mathcal{D} \partial_{x} \hat{J}, \quad \hat{J}=\hat{g} \circ \partial_{x} \hat{g}, \quad \hat{g}^{2}=\delta\left(t_{1}-t_{2}\right),
$$

and the equation for the Keldysh component $\hat{G}^{K}$

$$
\left[\hat{H}, \hat{G}^{K}\right]=i \mathcal{D} \partial_{x} \hat{J}^{K}, \quad \hat{J}^{K}=\hat{g}^{R} \circ \partial_{x} \hat{G}^{K}+\hat{G}^{K} \circ \partial_{x} \hat{g}^{A} .
$$

The boundary conditions for the functions $\hat{g}$ and $\hat{G}^{K}$ at the tunnel barrier follow from Eq. (4)

$$
\begin{gathered}
\hat{J}_{0}=\left(W / \xi_{0}\right)\left[\hat{g}_{-0}, \hat{g}_{+0}\right], \\
\hat{J}_{0}^{K}=\left(W / \xi_{0}\right)\left[\check{G}_{-0}, \check{G}_{+0}\right]^{K} .
\end{gathered}
$$

In Eqs. (8), the transparency parameter $W$ is defined as 


$$
W=R\left(\xi_{0}\right) / 2 R=\left(3 \xi_{0} / 4 \ell\right) \Gamma \gg \Gamma,
$$

where $R\left(\xi_{0}\right)=\xi_{0} / g_{N}$ is the normal resistance of the lead per length $\xi_{0}$. It has been shown in Refs. 11 and 12 that this quantity rather than the barrier transparency $\Gamma$ plays the role of a true transparency parameter in the theory of diffusive tunnel junctions (see also discussion in Sec. VII). In this paper, we will consider the limit $W \ll 1$, which corresponds to the conventional tunneling concept. In this case, according to Eqs. (8), the gradients of all functions are small. Within the tunnel model, which assumes $W$ to be the smallest parameter in the theory, these gradients are neglected, and the functions $\hat{g}$ and $\hat{f}$ are assumed to be local equilibrium within the leads. In our consideration, we will lift this assumption and suppose the local-equilibrium form of these functions only within the bulk electrodes (reservoirs). Attributing the reference point for the phase $\phi=0$ to the left electrode $x=$ $-L$, these functions in the right electrode $x=L$ are given by the relations

$$
\begin{gathered}
\hat{g}(E, t)=\sigma_{z} u_{S}\left(E+\sigma_{z} \mathrm{eV}\right)+i e^{i \sigma_{z} \phi(t)} \sigma_{y} v_{S}(E), \\
\left(u_{S}, v_{S}\right)^{R, A}=\frac{(E, \Delta)}{\left(E_{R, A}^{2}-\Delta^{2}\right)^{1 / 2}}, \quad E_{R, A}=E \pm i 0, \\
\hat{f}(E)=n_{\mathrm{eq}}\left(E+\sigma_{z} \mathrm{eV}\right), \quad n_{\mathrm{eq}}(E)=\tanh (E / 2 T),
\end{gathered}
$$

written in terms of the mixed Wigner representation $A(E, t)$ of the two-time functions

$$
A\left(t_{1}, t_{2}\right)=\int_{-\infty}^{+\infty} \frac{d E}{2 \pi} e^{-i E\left(t_{1}-t_{2}\right)} A(E, t) .
$$

In Eq. (10), the variable $E$ has the meaning of the quasiparticle energy, and $t=\left(t_{1}+t_{2}\right) / 2$ is a real time. Similar equations, with $\phi=0$ and $V=0$, apply to the left electrode $x=-L$.

Because of the small value of the tunneling parameter $W$ one can neglect the variation of the electric potential and the superconducting phase along the leads, and assume the voltage $V$ and the phase difference $\phi=2 \mathrm{eV} t$ between the reservoirs to be directly applied to the tunnel barrier. Following this argument, one can also neglect the variation of the charge imbalance function $f_{-}$proportional to a small electric field $(\sim \mathrm{eVW})$ penetrating the superconducting leads. Furthermore, the small value of the superfluid momentum in the superconducting leads, ${ }^{11,12} p_{s} \sim W / \xi_{0}$, enables us to neglect a small effect of the suppression of the energy gap, ${ }^{16}$ [ $\left.\sim\left(p_{s} \xi_{0}\right)^{4 / 3} \sim W^{4 / 3}\right]$, by the superfluid momentum. In such an approximation, the coefficients in Eq. (2) within the left lead $x<0$ are time-independent functions, similar to the value of $\breve{G}$ at the left electrode. At $x>0$, using the gauge transformation $\tilde{G}\left(x>0, t_{1}, t_{2}\right)=S^{\dagger}\left(t_{1}\right) \check{G}\left(x>0, t_{1}, t_{2}\right) S\left(t_{2}\right)$ of the function $\check{G}$ with a unitary operator $S(t)=\exp \left[i \sigma_{z} \phi(t) / 2\right],{ }^{17}$ we exclude the time-dependent phase and the electric potential from the equations for the function $\widetilde{G}$ and the boundary conditions (9) at $x=L$, which then become similar to the equations for $\breve{G}(x)$ at $x<0$ and the boundary conditions at $x=-L$. This results in the symmetry relation $\widetilde{G}(x)=\check{G}(-x)$, which allows us to replace the function $\check{G}_{+0}$ in the boundary condition (4) and in the expression (5) for the electric current by the inverse gauge transformation of the function $\check{G}_{0} \equiv \check{G}_{-0}$

$$
\check{G}_{+0} \rightarrow \overline{\check{G}}_{0} \equiv S\left(t_{1}\right) \check{\tilde{G}}_{+0} S^{\dagger}\left(t_{2}\right)=S\left(t_{1}\right) \check{G}_{0} S^{\dagger}\left(t_{2}\right) .
$$

As the result, the problem is reduced to the solution of a static equation for the function $\check{G}(x)$ within the left lead with the time-dependent boundary condition (4) at the tunnel barrier. A similar approach is used in the theory of ballistic point contacts, where the Josephson coupling is described by an effective time-dependent matching condition for the gaugetransformed Bogolyubov-de Gennes equations in the leads.

In a general nonstatic case, the function $\breve{G}\left(t_{1}, t_{2}\right)$ consists of a set of harmonics $\check{G}\left(E_{n}, E_{m}\right), E_{n}=E+n \mathrm{eV}$, which are coupled to each other through a complicated set of recursive equations following from Eqs. (2) (see discussion in Ref. 18). The problem essentially simplifies if the distance eV between the harmonics is much smaller than the smallest scale $\delta E$ of variations in the quasiparticle spectrum,

$$
\mathrm{eV} \ll \delta E \text {. }
$$

The magnitude of $\delta E$ will be indicated below, see Eq. (23).

\section{LOW VOLTAGE LIMIT}

In the limit of low applied voltages, the evolution of the quasiparticle spectrum and distribution function can be described within the adiabatic approximation using expansion over small Josephson frequency. In the static case $\mathrm{eV} \rightarrow 0$, the function $\check{G}$ depends only on the time difference $t_{1}-t_{2}$, and the Wigner transformation (10) reduces Eqs. (6) to the standard Usadel equations, ${ }^{19}$

$$
\begin{gathered}
E v-\Delta u=(i \mathcal{D} / 2) \partial_{x}\left(u \partial_{x} v-v \partial_{x} u\right), \\
u^{2}-v^{2}=1
\end{gathered}
$$

for the scalar components of the Green's function

$$
\hat{g}(x, E)=\sigma_{z} u+i \sigma_{y} v .
$$

The functions $u$ and $v$ determine the spectral characteristics of the system; in particular, the quantity $N(x, E)=\left(u^{R}\right.$ $\left.-u^{A}\right) / 2$ is the DOS normalized over its value $N_{F}$ in the normal state. In what follows, we will express the advanced Green's functions through the retarded ones $(u, v)^{A}=$ $-(u, v)^{R^{*}}$ using the general relation $\hat{g}^{A}=-\sigma_{z} \hat{g}^{R \dagger} \sigma_{z}$, and omit the superscript $R$, assuming all Green's functions to be retarded.

At small applied voltages, we proceed to the Wigner representation (10) of the two-time functions and expand the time convolutions in Eqs. (6)-(8) to first order in $\mathrm{eV}$

$$
(A \circ B)(E, t) \approx A B+(i / 2)\langle A, B\rangle,
$$

where $\langle A, B\rangle=\partial_{E} A \partial_{t} B-\partial_{t} A \partial_{E} B$ denotes the Poisson brackets in the energy-time space. Within such an approximation, the 
Green's function $\hat{g}(x, E, t)$ holds the matrix structure (14), and the gauge-transformed functions $\overline{\hat{g}}_{0}$ and $\bar{f}_{0}$ [see Eq. (11)], which enter the boundary conditions (8), read

$$
\begin{gathered}
\overline{\hat{g}}_{0}=\sigma_{z} u_{0}\left(E+\sigma_{z} \mathrm{eV}, t\right)+i e^{2 i \sigma_{z} \mathrm{eV} t} \sigma_{y} v_{0}(E, t), \\
\bar{f}_{0}=f_{0}\left(E+\sigma_{z} \mathrm{eV}, t\right) .
\end{gathered}
$$

The expression for the electric current obtained from Eqs. (5) and (15) consists of the three terms

$$
\begin{gathered}
I(t)=I_{1} \sin \phi+I_{2}+I_{3} \cos \phi, \\
I_{1}(t) \sin \phi=\frac{1}{2 e R} \int_{-\infty}^{\infty} d E I_{s}(E, t) f_{0}(E, t), \\
I_{2}(t)=\frac{V}{R} \int_{0}^{\infty} d E N_{0}^{2}(E, t) \partial_{E} f_{0}(E, t), \\
I_{3}(t)=\frac{V}{R} \int_{0}^{\infty} d E M_{0}^{2}(E, t) \partial_{E} f_{0}(E, t),
\end{gathered}
$$

where $f_{0}(E, t)$ is the boundary value of the distribution function $f(x, E, t)$, and $I_{s}=-\operatorname{Im} v_{0}^{2} \sin \phi, N_{0} \equiv N(x=0, E)=\operatorname{Re} u_{0}$, and $M_{0}=\operatorname{Re} v_{0}$ are the spectral densities of the different current components. Such structure of the current is similar to the result of the tunnel model: ${ }^{3}$ Indeed, when the current spectral densities and distribution function approach nonperturbed equilibrium form, the first term in Eq. (16a) describes the ac Josephson current, the term $I_{2}$ is the dissipative quasiparticle current which approaches the value $V / R$ in the normal state, and $I_{3}$ is the interference current. However, Eqs. (16) are more general; they include the Josephson oscillations of the spectral characteristics together with a nonequilibrium form of the distribution function.

\section{JUNCTION SPECTRUM}

To first order in $\mathrm{eV}$, the spectral functions $u(x, E, t)$ and $v(x, E, t)$ obey static Usadel Eqs. (13) with time-dependent boundary condition following from Eqs. (8a) and (15a)

$$
\xi_{0}\left(u \partial_{x} v-v \partial_{x} u\right)_{0}=-4 W(u v)_{0} \sin ^{2}(\phi / 2) .
$$

This boundary condition was found in Refs. 11 and 12 for a similar structure with the time-independent phase difference. Thus, at low enough voltages, the spectral characteristics of the junction adiabatically follow time variations of $\phi(t)$.

Equations (13) and (17) can be supplemented by helpful identities following from the unity components of the matrix Eqs. (6) and (8a)

$$
\begin{gathered}
\partial_{t} u=(i \mathcal{D} / 2) \partial_{x}\left(\left\langle u, \partial_{x} u\right\rangle-\left\langle v, \partial_{x} v\right\rangle\right), \\
\xi_{0}\left(\left\langle u, \partial_{x} u\right\rangle-\left\langle v, \partial_{x} v\right\rangle\right)_{0}=-W\left\langle v_{0}^{2}, \cos \phi\right\rangle .
\end{gathered}
$$

In order to satisfy the condition (13b), we introduce usual parametrization $u=\cosh \theta, v=\sinh \theta$. Furthermore, we will
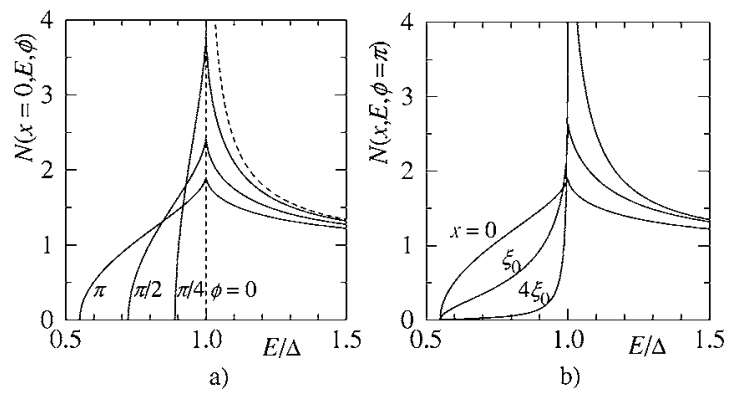

FIG. 2. Energy dependencies of the DOS for the transparency parameter $W=0.05$ : (a) at the tunnel barrier for different values of the superconducting phase; (b) at $\phi=\pi$ for different distances from the barrier.

neglect a small effect of suppression of the order parameter near the barrier, assuming $\Delta$ to be homogeneous [see comment to Eq. (23)]. Then the equation and the boundary condition for the spectral angle $\theta$, following from Eqs. (13a) and (17), take the form in a dimensionless variable $z=x / \xi_{0}$

$$
\begin{gathered}
\partial_{z} \theta=2 k \sinh \frac{\theta-\theta_{S}}{2}, \quad k(E)=\left[i \sinh \theta_{S}(E)\right]^{-1 / 2}, \\
\left(\partial_{z} \theta\right)_{0}=-2 \gamma(t) \sinh 2 \theta_{0}, \quad \gamma(t)=W \sin ^{2}[\phi(t) / 2] .
\end{gathered}
$$

The quantity $\gamma(t)$ in Eq. (20) has the meaning of a timedependent depairing factor related to the discontinuity of the superconducting phase $\phi(t)$ at the tunnel barrier. When the phase approaches a multiple of $2 \pi$, this factor turns to zero, and the spectral angle becomes homogeneous and equal to its bulk value $\theta_{S}(E)=\operatorname{arctanh}(\Delta / E)$. At arbitrary $\phi$, Eqs. (19) and (20) describe deviation of the spectral angle from $\theta_{S}$ at the distance $x \sim \xi_{0}$ from the barrier; thus, in a long junction $L \gg \xi_{0}$, we can apply the solution for a semi-infinite lead ${ }^{20}$

$$
\tanh \left[\left(\theta-\theta_{S}\right) / 4\right]=\tanh \left[\left(\theta_{0}-\theta_{S}\right) / 4\right] \exp (k z) .
$$

The equation for the boundary value $\theta_{0}(E, t)$ of the spectral angle follows from Eqs. (19)-(21)

$$
\sinh \frac{\theta_{S}(E)-\theta_{0}(E, t)}{2}=\frac{\gamma(t)}{k(E)} \sinh 2 \theta_{0}(E, t) .
$$

The behavior of the DOS calculated by the numerical solution of Eq. (22) is shown in Figs. 2 and 3. At $\phi=0$ (we consider the phase within the period $0 \leqslant \phi<2 \pi$ ), the DOS approaches the BCS energy dependence depicted by the dashed line in Fig. 2(a). The current through the junction affects the singularity of DOS at the bulk energy gap, which becomes a beak-shaped peak with root singularities of the derivatives resulting from the divergence of the decay length $k^{-1}(E)$ at $E \rightarrow \Delta$ [see Eq. (19)]. This divergence ${ }^{20}$ is an ana$\log$ of the long-range proximity effect at $E \rightarrow 0$ in NS structures (see, e.g., a review ${ }^{21}$ ). The DOS identically turns to zero inside an oscillating minigap at $E<E_{*}(t)<\Delta$. Similar conclusions can be drawn regarding behavior of the current spectral densities shown in Figs. 4(a)-4(c).

Within the subgap region, $E_{*}(t)<E<\Delta$, the DOS decreases at the distance $\gtrsim \xi_{0}$ from the barrier (more precisely, 


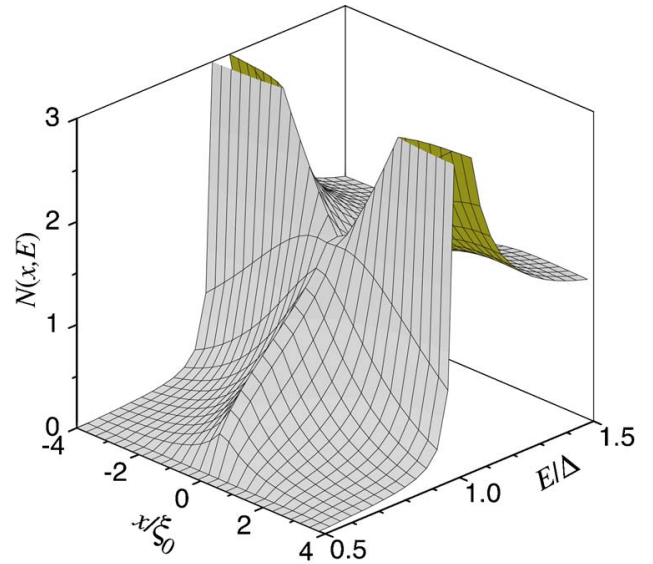

FIG. 3. (Color online) 2D profile of the DOS in the vicinity of the tunnel barrier at $W=0.05$, at the moments of maximum depth of the Andreev band $(\phi=\pi)$.

at $\xi_{0}|E / \Delta-1|^{-1 / 4}$, due to the long-range proximity effect mentioned above), as shown in Fig. 2(b). This implies that the subgap states form an oscillating cluster of bound states "Andreev band," which has a triangular shape in the $(E, x)$-space as shown in Fig. 3 (similar cluster appears near the lower gap edge $E=-\Delta$ ). The energy depth of the cluster is

$$
\delta E=\Delta-E_{*} \approx 6 \Delta W^{4 / 5} \sin ^{8 / 5}(\phi / 2),
$$

[see Eq. (28) below], and it spreads over the distance of several $\xi_{0}$ from the tunnel barrier. We note that because of comparatively large value of $\delta E$, we may neglect in the spec-

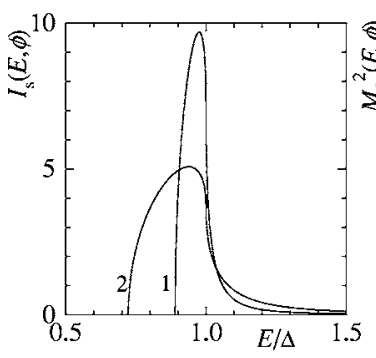

a)

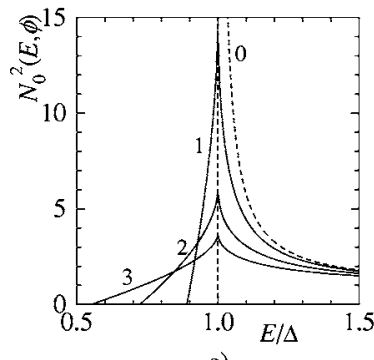

c)

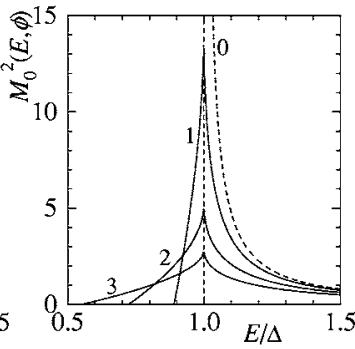

b)

d)

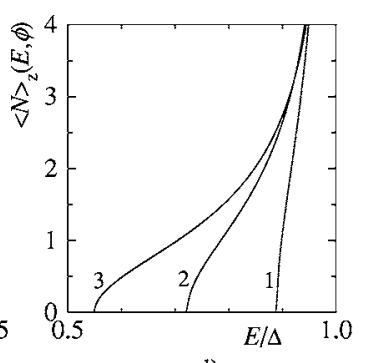

FIG. 4. Spectral characteristic of the junction at $W=0.05$, for different values of the superconducting phase: $\phi=0$ (curves indicated by 0 ), $\pi / 4$ (1), $\pi / 2$ (2), and $\pi$ (3). (a)-(c): spectral densities $I_{s}, M_{0}^{2}$, and $N_{0}^{2}$ of the Josephson, interference, and quasiparticle currents, respectively; (d): spatially averaged DOS of the bound states. tral characteristics a smaller $(\sim \Delta W)$ effect of local suppression of the order parameter $\Delta$ in the vicinity of the barrier. ${ }^{12}$ Obviously, the quantity $\delta E$ plays the role of the smallest characteristic energy of the spectrum in Eq. (12).

The small value of the parameter $W$ enables us to apply a perturbative approach for solving Eq. (22). At the energies far enough from the gap edge, where $\theta$ is of the order of unity, the quantity $\theta_{0}$ is close to $\theta_{S}$, and Eq. (22) leads to the following asymptotic relation for $\theta_{0}$ :

$$
\cosh \theta_{0}=\cosh \theta_{S}-2 \gamma(t) \sinh 2 \theta_{S}\left(i \sinh ^{3} \theta_{S}\right)^{1 / 2} .
$$

However, when the energy approaches $\Delta$, this expansion fails due to the divergence of $\theta_{S}$ at the bulk gap edge. This requires modification of the perturbative theory within the region $|E-\Delta| \ll \Delta,{ }^{12}$ where the quantities $\theta_{0}$ and $\theta_{S}$ are large, while their difference $\theta_{0}-\theta_{S}$ may have an arbitrary value. Using these arguments, we hold only large exponents $\exp \theta_{0, S}$ in the hyperbolic functions in the right-hand side (rhs) of Eq. (22) and use the asymptotic expression $\exp \theta_{S}$ $\approx \sqrt{2 \Delta /(E-\Delta)}$ at small $|E-\Delta|$. Then, introducing a dimensionless energy variable $\varepsilon$ and the normalized spectral function $y(\varepsilon)$ according to relations

$$
\begin{gathered}
\varepsilon=p^{2}(t)(E-\Delta) / 2 \Delta, \quad p(t)=\left[2 / \gamma^{2}(t)\right]^{1 / 5}, \\
\exp \theta_{0}=p(t) y(\varepsilon), \quad \exp \theta_{S}=p(t) \varepsilon^{-1 / 2},
\end{gathered}
$$

we reduce Eq. (22) to a numerical algebraic equation for $y(\varepsilon)$

$$
(y \sqrt{\varepsilon}-1)^{2}=i y^{5} .
$$

According to Eqs. (25), the parameter $p(t) \sim \delta E^{-1 / 2}$ determines a characteristic scale of the spectral functions $u_{0}, v_{0}$ $\approx(1 / 2) \exp \theta_{0}$ in the vicinity of the bulk gap edge.

The choice of the relevant complex root $y(\varepsilon)$ of Eq. (26) is determined by the requirement for the asymptotic behavior at $\varepsilon \gg 1$, which must coincide with the energy dependence given by direct perturbative expansion (24) at $\delta E \ll|E-\Delta|$ $\ll \Delta$. Within the main approximation, the function $y(\varepsilon)$ turns to zero at large $\varepsilon$ as $\varepsilon^{-1 / 2}$. At the energies $\varepsilon$ smaller than $\varepsilon_{*}=-(25 / 6)(2 / 3)^{1 / 5} \approx-3.84$, the function $y(\varepsilon)$ becomes imaginary, and therefore the spectral functions

$$
N_{0}=M_{0}=\frac{1}{2} p(t) \operatorname{Re} y, \quad I_{s}=-\frac{1}{4} p^{2}(t) \operatorname{Im} y^{2} \sin \phi,
$$

turn to zero at $E<E_{*}(t)$, where the minigap edge $E_{*}(t)$ is determined by the relation following from Eq. (25a)

$$
E_{*}(t)=\Delta\left[1-C \gamma^{4 / 5}(t)\right], \quad C=25 /\left(3 \cdot 6^{1 / 5}\right) \approx 5.82 .
$$

We note that due to a moderately small value $W=0.05$ of the transparency parameter, the minigap values, obtained by the numerical solution of Eq. (22) and shown in Figs. 2 and 4, differ slightly from their approximate values found from Eq. (28) (by some 10\%). Using the improved perturbation theory, IPT) approximation, however, significantly simplifies both the analytical and numerical calculations, allowing us to factorize the time and energy dependencies of the spectral func- 
tions. Applying this approximation to Eq. (21), we obtain the spatial dependence of the spectral angle

$$
\begin{gathered}
\exp \theta(x, E, t)=p(t) \varepsilon^{-1 / 2} \tanh ^{2}[s(\varepsilon)-k(\varepsilon, t) z / 2], \\
\tanh ^{2} s(\varepsilon)=y(\varepsilon) \sqrt{\varepsilon}, \quad k(\varepsilon, t)=[2 \sqrt{\varepsilon} / i p(t)]^{1 / 2} .
\end{gathered}
$$

It is interesting to note that the spectral functions are inhomogeneous within the leads at $E=\Delta$ and finite at any finite distance from the barrier, as it is seen in Fig. 3, which seems to contradict the divergence of the characteristic decay length $k^{-1}(E)$ of the spectral angle at the gap edge. The explanation to this effect is the following: At $E \rightarrow \Delta(\varepsilon \rightarrow 0)$, the functions $s(\varepsilon)$ and $k(\varepsilon, t)$ in Eq. (30) turn to zero as $\varepsilon^{1 / 4}$, which cancels the divergence of the prefactor in Eq. (29) and results in the following expression for the spectral angle at the gap edge:

$$
\exp \theta(x, \Delta, t)=p(t) y(0)[1-z / \sqrt{2 i p(t) y(0)}]^{2} .
$$

According to Eq. (31), the spectral functions at $E=\Delta$ and $z$ $\rightarrow \infty$ diverge, which restores their BCS divergence at the gap edge within the bulk superconductor.

\section{DISTRIBUTION FUNCTION}

To first order in eV, Eq. (7) in the Wigner representation has the form of a diffusive kinetic equation ${ }^{13}$

$$
N \partial_{t} f=\mathcal{D} \partial_{x}\left(D_{+} \partial_{x} f\right),
$$

where $D_{+}=(1 / 2)\left(1+|u|^{2}-|v|^{2}\right)$ is the dimensionless diffusion coefficient. At the reservoir, the distribution function approaches equilibrium value, $f(-L)=n_{\mathrm{eq}}(E)$. The boundary condition at the tunnel barrier following from Eq. (8b)

$$
\left(D_{+} \partial_{x} f\right)_{0}=\beta \partial_{E} f_{0},
$$

determines the boundary value of the flow $D_{+} \partial_{x} f$ of nonequilibrium quasiparticles escaping from the barrier. In this equation, the function

$$
\beta(E, t)=\mathrm{eV} I_{s}(E, t) W / \xi_{0}
$$

describes the source of the nonequilibrium-the energy exchange between the quasiparticles and the superfluid condensate due to the oscillations the supercurrent spectral density $I_{s}$. This results in the DOS oscillations which can be expressed through $I_{s}$ by integrating the identity (18a) over the left lead, taking into account Eq. (18b) and extending the integration over the whole negative semiaxis due to the rapid decay of the time derivatives of all quantities at a small distance $x \sim \xi_{0}$

$$
\left\langle\partial_{t} N\right\rangle_{x}(E, t) \equiv \int_{-\infty}^{0} \partial_{t} N(x, E, t) d x=\mathcal{D} \partial_{E} \beta(E, t) .
$$

Due to the existence of the oscillating Andreev band in the vicinity of the barrier, the behavior of the quasiparticles strongly depends on their energy. Indeed, most of excitations with the energy $E>\Delta$ spend a short time $\sim \Delta^{-1}$ near the barrier, they rapidly diffuse away along the leads and escape into the reservoirs (traveling quasiparticles). The quasiparticles with low energy approaching the contact at the distance $\sim \xi_{0}$ while the depth of the Andreev band increases $(0<\phi$ $<\pi)$ are trapped by the Andreev band and spend a much larger time $\sim(\mathrm{eV})^{-1}$ inside the band, following the spectrum oscillations. During the next half-period $(\pi<\phi<2 \pi)$, the depth of the Andreev band decreases, and the trapped quasiparticles are pushed out to the extended states. Such a physical picture is similar to the quasiparticle dynamics within the surface skin layer of a superconductor irradiated by rf electromagnetic field. ${ }^{22}$ Below we will perform a separate analysis of the kinetics of the traveling and trapped quasiparticles.

\section{A. Traveling quasiparticles}

At $E>\Delta$, the coefficients $N$ and $D_{+}$in Eq. (32) rapidly vary in the near vicinity $x \sim \xi_{0}$ of the barrier and then, at $x$ $\gg \xi_{0}$, approach their values in a bulk superconductor $N$ $\rightarrow N_{S}(E)=E / \sqrt{E^{2}-\Delta^{2}}, D_{+} \rightarrow 1$. Correspondingly, the solution of Eq. (32) has the form of a slowly varying function $f^{(0)}$ with a small but rapidly varying addition $f^{(1)}$, which vanishes at the distances $x \gg \xi_{0}$ from the barrier. Thus, within the main approximation, the population of the extended states, $f_{>}$ $=f^{(0)}(x, E>\Delta, t)$, satisfies Eq. (32) with the asymptotic values of the coefficients at $x \gg \xi_{0}$

$$
N_{S} \partial_{t} f_{>}=\mathcal{D} \partial_{x}^{2} f_{>}
$$

To derive an effective boundary condition to this equation, we subtract Eq. (36) from the initial kinetic Eq. (32), keeping a large spatial derivative of the function $f^{(1)}$

$$
\left(N-N_{S}\right) \partial_{t} f_{>}=\mathcal{D} \partial_{x}\left[\left(D_{+}-1\right) \partial_{x} f_{>}+D_{+} \partial_{x} f^{(1)}\right],
$$

and then integrate Eq. (37) over $x$ along the left lead. Since all terms in this equation vanish at $x \gg \xi_{0}$, we extend the integration over the whole semiaxis, similar to Eq. (35), and take the smooth function $f_{>}$in its left-hand side (lhs) at $x$ $=0$. Then, using Eq. (33), we obtain the boundary condition

$$
\left\langle N-N_{S}\right\rangle_{x}\left(\partial_{t} f_{>}\right)_{0}=\mathcal{D}\left(\beta \partial_{E} f_{>}-\partial_{x} f_{>}\right)_{0} .
$$

The distribution function $f_{>}$consists of a static part $g(x, E)$ and a dynamic (time-dependent) part $f_{>}(x, E, t)$ $=g(x, E)+h(x, E, t)$. The static part linearly varies along the lead and approaches equilibrium distribution function $n_{\text {eq }}(E)$ at $x=-L$

$$
g(x, E)=g_{0}(E)+(x / L)\left[g_{0}(E)-n_{\mathrm{eq}}(E)\right] .
$$

The dynamic component $h(x, E, t)$ has the form

$$
\begin{gathered}
h(x, E, t)=\sum_{n} h_{n}(E) e^{-i n \mathrm{eV} t+K_{n} x}, \quad\langle h\rangle_{t}=0, \\
K_{n}(E)=\sqrt{|n| \mathrm{eV} N_{S}(E) \mathcal{D}^{-1}} \exp [-(i \pi / 4) \operatorname{sgn} n] .
\end{gathered}
$$

Here $\langle\ldots\rangle_{t}$ denotes time averaging over the period $\tau$ $=2 \pi / \mathrm{eV}$, and $h_{n}(E)$ are the Fourier harmonics of the function $h(0, E, t)$

$$
h_{n}(E)=\int_{0}^{\tau} \frac{d t}{\tau} h(0, E, t) e^{i n \mathrm{eV} t} .
$$

Equation (40) was obtained assuming the decay length $L_{V}$ $=K_{n}^{-1} \sim \sqrt{\mathcal{D} / \mathrm{eV}}$ of the oscillations of the distribution function 
to be much smaller than the junction length $L$; this enables us to apply the solution for a semi-infinite lead. In the opposite case $L_{V} \gg L$, the reservoirs effectively suppress these oscillations, and the function $h$ rapidly decays while the voltage decreases. The equation for the harmonics $h_{n}(E)$ follows from Eq. (38)

$$
\begin{gathered}
\mathcal{D}^{-1}\left\langle N-N_{S}\right\rangle_{x} \partial_{t} h(0, E, t)-\beta \partial_{E} h(0, E, t)+\left(\partial_{x} g\right)_{0} \\
+\sum_{n} h_{n}(E) K_{n} e^{-i n \mathrm{~V} t}=\beta \partial_{E} g_{0}(E) .
\end{gathered}
$$

The first two terms in the lhs of Eq. (41), proportional to a small applied voltage $\mathrm{eV}$, can be neglected as compared to the fourth term. Then, averaging Eq. (41) over $t$ and using the identity (35), we obtain the expression for the third term

$$
\left(\partial_{x} g\right)_{0}=\partial_{E}\langle\beta h\rangle_{t},
$$

which therefore is also proportional to small $\mathrm{eV}$ and can be neglected as well. As the result, the approximate solution of Eq. (41) reads $h_{n}=\beta_{n} K_{n}^{-1} \partial_{E} g_{0}$, i.e., the dynamic part $h(x, E, t)$ of the distribution function is expressed through the boundary value of the static part $g_{0}(E)$.

It follows from this consideration that the dynamic part of the distribution is generated by the oscillations of the quasiparticle spectrum at $E>\Delta$. These oscillations provide the energy transfer from the electric field to quasiparticles, which generally results in the "heating" of quasiparticles, i.e., their redistribution toward higher energies (pumping effect). The heating is described by a diffusion equation for $g_{0}$ in the energy space following from Eqs. (42) and (39)

$$
\begin{gathered}
\partial_{E}\left(D_{E} \partial_{E} g_{0}\right)=L\left(\partial_{x} g\right)_{0}=g_{0}-n_{\mathrm{eq}}, \\
D_{E}=L \sum_{n}\left|\beta_{n}\right|^{2} K_{n}^{-1} .
\end{gathered}
$$

A similar equation has been obtained in Refs. 23 for multiple Andreev-reflection (MAR)-induced heating in long SNS junctions, where the nonequilibrium is constrained by inelastic collisions. In our strongly inhomogeneous case, the role of the relaxation factor in Eq. (43) is played by the diffusive flow $\left(\partial_{x} g\right)_{0}$ of nonequilibrium quasiparticles from the junction. The intensity of the heating effect is determined by the estimate of the diffusion term in Eq. (43) with the equilibrium function $n_{\text {eq }}, \partial_{E}\left(D_{E} \partial_{E} n_{\mathrm{eq}}\right) \sim\left(L / \xi_{0}\right) W(\mathrm{eV} / \delta E)^{3 / 2}$. Due to the presence of the two small factors, this term is small for reasonable lengths of the leads, which enables us to approximate the function $g_{0}$ with the equilibrium distribution function $g_{0} \approx n_{\mathrm{eq}}$. Then the boundary value of the distribution function of traveling quasiparticles reads

$$
\begin{gathered}
f_{>}(0, E, t)=n_{\mathrm{eq}}(E)+h(0, E, t), \\
h(0, E, t)=n_{\mathrm{eq}}^{\prime} \sum_{n} \beta_{n} K_{n}^{-1} e^{-i n \mathrm{eV} t}, \quad n_{\mathrm{eq}}^{\prime} \equiv \partial_{E} n_{\mathrm{eq}}(E) .
\end{gathered}
$$

\section{B. Trapped quasiparticles}

At small voltages $\mathrm{eV} \ll \Delta$, the spatial size $\xi_{0}$ of the Andreev band is much smaller than the smallest kinetic length
$L_{V}$, therefore the main part $f^{(0)}$ of the distribution function of the trapped quasiparticles is spatially uniform $f_{<} \approx f^{(0)}(E$ $<\Delta, t)$. Then the kinetic Eq. (32) takes the form

$$
N \partial_{t} f_{<}=\mathcal{D} \partial_{x}\left(D_{+} \partial_{x} f^{(1)}\right)
$$

By averaging Eq. (46) over $x$ and using the boundary condition (33), we obtain a partial differential equation (PDE) for the function $f_{<}$

$$
\langle N\rangle_{x} \partial_{t} f_{<}-\mathcal{D} \beta \partial_{E} f_{<}=0 .
$$

As long as the coefficients of this PDE satisfy the identity (35), the equation $d \xi=\langle N\rangle_{x} d E+\mathcal{D} \beta d t=0$ for its characteristic curves $\xi(E, t)=$ const in the $(E, t)$ space is easily integrated

$$
\xi(E, t)=\xi_{0}^{-1} \int_{E_{*}(t)}^{E} d E^{\prime}\left\langle N\left(x, E^{\prime}, t\right)\right\rangle_{x} .
$$

This allows us to reduce the PDE (47) to the ordinary differential equation for the function $f_{<}$along the characteristics

$$
\left.\partial_{t} f_{<}(\xi, t)\right|_{\xi=\text { const }}=0 .
$$

The quantity $\xi(E, t)$ has the meaning of an averaged number of states with the energy smaller than given energy $E$, normalized over $N_{F}$; in the bulk superconductor, it approaches $\Theta(E-\Delta) \sqrt{E^{2}-\Delta^{2}}$. The bottom $E_{*}(t)$ of the Andreev band represents the reference point, $\xi\left[E_{*}(t), t\right]=0$. Within the (IPT) approximation, Eq. (48) reads

$$
\begin{gathered}
\xi(\varepsilon, t)=\Delta[4 \gamma(t)]^{1 / 5} J(\varepsilon), \quad J(\varepsilon)=\int_{\varepsilon_{*}}^{\varepsilon} d \varepsilon^{\prime} n\left(\varepsilon^{\prime}\right), \\
n(\varepsilon)=|\varepsilon|^{-1 / 2} \operatorname{Im} \sqrt{i y(\varepsilon)},
\end{gathered}
$$

where the function $n(\varepsilon)$ is related to the averaged DOS as $\langle N\rangle_{z}=\left[2 \gamma^{3}(t)\right]^{-1 / 5} n(\varepsilon)$. As follows from Eq. (50b), the function $\langle N\rangle_{z}(E)$ plotted in Fig. 4(d) exhibits a singularity at the bulk gap edge, due to the long-range proximity effect in the superconductor [see comments to Eq. (22)].

According to Eq. (49), the distribution of trapped excitations is a function of the "integral of motion" $\xi(E, t)$

$$
f_{<}(E, t)=f[\xi(E, t)],
$$

and therefore holds a constant value along the trajectories $\xi(E, t)=$ const in the $(E, t)$ space shown in Fig. 5. These trajectories can be interpreted as a diffusive analog of Andreev levels adiabatically moving in a slowly varying external field (cf. Ref. 24). From this we conclude that the trapped quasiparticles are completely dragged by the spectrum oscillations, and their energy periodically changes, in accordance with the relation following from Eqs. (48) and (35)

$$
\left.\partial_{t} E\right|_{\xi=\text { const }}=-\mathcal{D} \beta\langle N\rangle_{x}^{-1} .
$$

Since the bound states at the tunnel barrier appear and vanish periodically, their population is imposed by the distribution of traveling quasiparticles at the bulk gap edge. This is expressed by the matching condition 


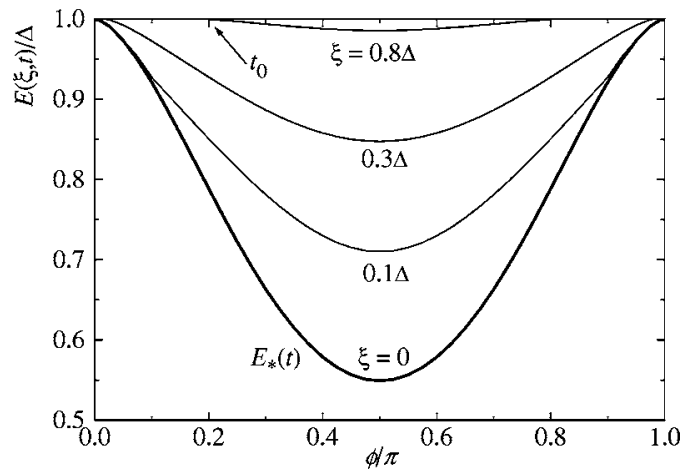

FIG. 5. Levels of equal number of states $\xi(E, t)=$ const versus phase difference at $W=0.05$. Bold line depicts the edge $E_{*}(t)$ $=E(\xi=0, t)$ of the minigap, and $t_{0}$ indicates a particular moment of trapping to the trajectory $\xi=0.8 \Delta$.

$$
f_{<}(\xi)=f_{>}\left[0, \Delta, t_{0}(\xi)\right], \quad 0<\phi<\pi .
$$

Here $t_{0}(\xi)$ is the moment of crossing the gap edge by the quasiparticle with the given value of $\xi$ (see Fig. 5), i.e., it is the smallest solution of the equation $E\left(\xi, t_{0}\right)=\Delta$ within the period of the DOS oscillations.

Since the trapped quasiparticles spend a long time at the barrier, the heating effect for them becomes well pronounced, in contrast to the traveling quasiparticles. Indeed, as follows from Eq. (45), the time-dependent part $h$ of the distribution function turns to zero at $E=\Delta$ due to the singularity of $N_{S}$, and therefore the distribution function of trapped quasiparticles turns into a plateau (i.e., does not depend on energy)

$$
f_{<}(\xi)=g_{0}(\Delta)=n_{\text {eq }}(\Delta) .
$$

Nonadiabatic correction to the distribution function (54) is produced by inelastic relaxation. To include the effect of inelastic collisions on the quasiparticle kinetics, we will add a collision term in the $\tau$ approximation $N \tau_{\varepsilon}^{-1}\left[n_{\mathrm{eq}}-f\right]$, to the rhs of the kinetic Eq. (46). Within such a model, the kinetic equation for the trapped quasiparticles reads

$$
\partial_{t} f_{<}(\xi, t)=\tau_{\varepsilon}^{-1}\left\{n_{\mathrm{eq}}[E(\xi, t)]-f_{<}(\xi, t)\right\} .
$$

The initial condition to Eq. (55) follows from Eq. (53)

$$
f_{<}\left(\xi, t_{0}\right)=n_{\mathrm{eq}}(\Delta)
$$

\section{ELECTRIC CURRENT}

Proceeding to the calculation of the electric current (16a), we start from the analysis of the ac Josephson current $I_{1} \sin \phi$. First, we note that the effect of the DOS oscillations on the amplitude $I_{1}$, calculated with the equilibrium distribution function $n_{\text {eq }}$, gives rise only to small corrections to the tunnel model result, $\left.I_{1}^{\mathrm{eq}}=(\pi \Delta / 2 e R) \tanh (\Delta / 2 T)\right)^{2,3}$ Indeed, in this case, the integral in Eq. (16b) is determined by the imaginary Matsubara energies $i \omega_{n}=\pi i T(2 n+1)$ far from the gap edges, which allows us to apply ordinary perturbation approach [see Eq. (24)] to the calculation of the spectral density $I_{s}$. This results in the correction of the order of $W$ to $I_{1}^{\mathrm{eq}},{ }^{12}$ which will be neglected below.
In what follows, we will focus on the nontrivial contributions of nonequilibrium quasiparticles to the Josephson current $I_{1}$. Such contributions, $I_{h}(t)$ and $I_{<}(t)$, come from the dynamic part $h$ of the distribution of traveling quasiparticles and the distribution $f_{<}$of trapped quasiparticles

$$
\begin{aligned}
& I_{h}(t)=\frac{1}{e R} \sum_{n \neq 0} e^{-i n \mathrm{eV} t} \int_{\Delta}^{\infty} d E I_{s} K_{n}^{-1} \beta_{n} n_{\mathrm{eq}}^{\prime}(E), \\
& I_{<}(t)=\frac{1}{e R} \int_{E_{*}}^{\Delta} d E I_{s}\left\{f_{<}[\xi(E, t), t]-n_{\mathrm{eq}}(E)\right\} .
\end{aligned}
$$

Due to rapid convergence of the integral in Eq. (57a) at $E-\Delta \lesssim \delta E \ll(\Delta, T)$, the function $n_{\text {eq }}^{\prime}(E)$ can be taken at $E$ $=\Delta$. For a similar reason, we apply the IPT expression (27) for the spectral density $I_{s}$, which leads to the following result:

$$
I_{h}(t)=\frac{\Delta F_{1}}{e R} \sqrt{\frac{\mathrm{eV}}{2 \Delta}} W^{2 / 5} P(t) \sin \phi,
$$

where

$$
\begin{gathered}
F_{1}(T)=\Delta n_{\mathrm{eq}}^{\prime}(\Delta)=\frac{\Delta}{2 T} \cosh ^{-2} \frac{\Delta}{2 T}, \\
P(t)=C_{1} \sin ^{2 \nu} \frac{\phi}{2} \sum_{m=1}^{\infty} \frac{(-1)^{m} \sqrt{m} \sin (m \phi-\pi / 4)}{\left(m^{2}-\nu^{2}\right) B(\nu+m, \nu-m)}, \\
C_{1}=2^{1-\nu / 2} \int_{0}^{\infty} d \varepsilon \varepsilon^{1 / 4}\left[\operatorname{Im} y^{2}(\varepsilon)\right]^{2} \approx 0.15, \quad \nu=0.2,
\end{gathered}
$$

and $B(x, y)$ is the Euler's beta function. The time average of the current (58) does not vanish

$$
\begin{gathered}
I_{h}^{\mathrm{dc}} \equiv\left\langle I_{h}\right\rangle_{t}=\frac{\Delta F_{1}}{e R} C_{3} W^{2 / 5} \sqrt{\frac{\mathrm{eV}}{2 \Delta}}, \\
C_{3}=\langle P(t) \sin \phi\rangle_{t}=0.018,
\end{gathered}
$$

and, moreover, it exhibits a strongly nonlinear $(\sim \sqrt{V})$ voltage dependence. We recall that the validity of these results is restricted by the condition of small diffusion length as compared to the junction length $L_{V}=\sqrt{\mathcal{D} / \mathrm{eV}} \ll L$, or, equivalently, $\mathrm{eV} \gg E_{\mathrm{Th}}$, where $E_{\mathrm{Th}}=\mathcal{D} / L^{2}$ is the Thouless energy [see comments to Eq. (40)]. In the opposite case $\mathrm{eV} \ll E_{\mathrm{Th}}$, the oscillations of the distribution function are damped by equilibrium reservoirs, and the nonequilibrium dc current, produced by traveling quasiparticles, rapidly vanishes. A similar damping effect is caused by inelastic collisions, when the inelastic scattering length becomes smaller than the diffusion length $L_{V}$.

The dissipative dc current of traveling quasiparticles results from nonadiabatic (diffusive) evolution of the distribution function $h(t)$. A similar conclusion is also true for the trapped quasiparticles: Only the nonadiabatic part of the distribution function contributes to the dissipative dc current. Indeed, inserting the adiabatic distribution function $n_{\mathrm{eq}}(\Delta)$, Eq. (54), into Eq. (57b), and taking advantage of a small 
integration interval, which allows us to expand the difference of the equilibrium functions over $E-\Delta$, we get

$$
\begin{aligned}
& I_{<}^{\mathrm{ad}}(t)=\frac{\Delta F_{1}}{e R} C_{2} W^{4 / 5} \sin ^{8 / 5} \frac{\phi}{2} \sin \phi, \\
& C_{2}=2^{-2 \nu} \int_{\varepsilon_{*}}^{0} d \varepsilon \varepsilon \operatorname{Im} y^{2}(\varepsilon) \approx 3.11 .
\end{aligned}
$$

This equation contains only odd harmonics of the ac Josephson current. The dc current results from the nonadiabatic correction $\delta f$ to the distribution function, found from the solution of the kinetic Eq. (55)

$$
I_{<}^{\mathrm{dc}}=\frac{1}{e R}\left\langle\int_{E_{*}}^{\Delta} d E I_{s} \delta f[\xi(E, t), t]\right\rangle_{t}=\frac{\Delta F_{1}}{e R} W^{4 / 5} K(\mathrm{eV}) .
$$

The factor $K(\mathrm{eV})$ in Eq. (61) is a complicated numerical function of the relaxation parameter $\mathrm{eV} \tau_{\varepsilon}$. In the limits of weak $\left(\mathrm{eV} \tau_{\varepsilon} \gg 1\right)$ and strong $\left(\mathrm{eV} \tau_{\varepsilon} \ll 1\right)$ relaxation, this function decreases as $\left(\mathrm{eV} \tau_{\varepsilon}\right)^{-1}$ and $\mathrm{eV} \tau_{\varepsilon}$, respectively. In the spirit of our modeling approach to the problem of inelastic scattering, it is reasonable to approximate $K(\mathrm{eV})$ by the usual relaxation factor

$$
K(\mathrm{eV}) \approx \frac{\alpha_{1} \mathrm{eV} \tau_{\varepsilon}}{\alpha_{2}+\left(\mathrm{eV} \tau_{\varepsilon}\right)^{2}},
$$

where the parameters $\alpha_{1,2}$ are to be evaluated from the asymptotics of $K(\mathrm{eV})$ in both limiting cases.

In the weak relaxation limit $\mathrm{eV} \tau_{\varepsilon} \gg 1$ the adiabatic distribution function is given by Eq. (54), $f_{<}^{\mathrm{ad}}=n_{\mathrm{eq}}(\Delta)$; then the nonadiabatic correction $\delta f$ is determined by the local energy $E(\xi, t)$ averaged along the trajectories $\xi=$ const

$$
\delta f(\xi, t)=\tau_{\varepsilon}^{-1} n_{\mathrm{eq}}^{\prime}(\Delta) \int_{t_{0}(\xi)}^{t} d t^{\prime}\left[E\left(\xi, t^{\prime}\right)-\Delta\right] .
$$

Substituting $\delta f$ into the expression (61) and using the IPT relations $(50)$ for the function $\xi(E, t)$, we obtain the coefficient $\alpha_{1}$ in the relaxation factor $K(\mathrm{eV})$

$$
\alpha_{1}=\frac{B(0.5,2.3)}{2^{2 / 5} \pi} \int_{\varepsilon_{*}}^{0} d \varepsilon \operatorname{Im} y^{2}(\varepsilon) \int_{0}^{1} d x \varepsilon_{0}\left[x^{1 / 5} J(\varepsilon)\right] \approx 1.38,
$$

where $\varepsilon_{0}(\zeta)$ is the solution of equation $\zeta=J\left(\varepsilon_{0}\right)$, and the function $J(\varepsilon)$ is defined in Eq. (50a).

In the opposite limit of strong relaxation $\mathrm{eV} \tau_{\varepsilon} \ll 1$, the initial condition (56) is quickly "forgotten," and the adiabatic part of the distribution is the equilibrium function of the local energy, $f_{<}^{\text {ad }}=n_{\text {eq }}[E(\xi, t)]$. In this case, the nonadiabatic addition $\delta f$ is proportional to the time derivative of $E(\xi, t)$ along the trajectory

$$
\delta f(\xi, t) \approx-\tau_{\varepsilon} n_{\mathrm{eq}}^{\prime}(\Delta) \partial_{t} E(\xi, t) .
$$

Using the expression (52) for $\partial_{t} E$ and calculating $I_{<}^{\mathrm{dc}}$ in Eq. (61) in the IPT approximation, we arrive at the following relation between the coefficients $\alpha_{1}$ and $\alpha_{2}$ :

$$
\frac{\alpha_{1}}{\alpha_{2}}=\int_{0}^{\pi} \frac{d \tau \sin ^{2} 2 \tau}{2^{7 / 5} \pi \sin ^{2 / 5} \tau} \int_{\varepsilon_{*}}^{0} d \varepsilon \sqrt{|\varepsilon|} \frac{\left[\operatorname{Im} y^{2}(\varepsilon)\right]^{2}}{\operatorname{Im} \sqrt{i y(\varepsilon)}} \approx 1.74 .
$$

Now we turn to evaluation of the quasiparticle current $I_{2}$ and the interference current $I_{3} \cos \phi$. These currents are proportional to a small applied voltage, and therefore they can be calculated within the equilibrium approximation for the quasiparticle distribution neglecting small nonequilibrium correction. The contribution of the trapped quasiparticles is small, because of the small occupied phase volume, and can be neglected. Moreover, in the limit of weak relaxation, this contribution identically turns to zero because of an energyindependent distribution $f_{<}$. Within the BCS approximation for the spectral functions $N_{0}$ and $M_{0}$, the integrals in Eqs. (16c) and (16d) logarithmically diverge at the gap edge. The effect of the phase difference on the quasiparticle spectrum eliminates this divergence and effectively cuts the spectral functions at the value $(\delta E)^{-1 / 2}$ [see comments to Eq. (25)], which is equivalent to the effective cutoff in energy $E-\Delta$ $\gtrsim \delta E$. Using the IPT expressions (27) for the spectral functions at $E-\Delta \ll \Delta$, we obtain to the main order in $W$

$$
\begin{gathered}
I_{2}(t)=I_{3}(t)+\frac{V}{R}\left[1-n_{\mathrm{eq}}(\Delta)\right], \\
I_{3}(t)=\frac{V}{R}\left[F_{1} \ln \sqrt{a / \gamma^{4 / 5}(t)}-F_{2}\right],
\end{gathered}
$$

where

$$
\begin{gathered}
F_{2}(T)=\int_{\Delta}^{\infty} \frac{\Delta^{2} d E}{E^{2}-\Delta^{2}}\left[n_{\mathrm{eq}}^{\prime}(\Delta)-n_{\mathrm{eq}}^{\prime}(E)\right], \\
\ln a=\int_{0}^{\infty} d \varepsilon\left[\operatorname{Re}^{2} y(\varepsilon)-\frac{\Theta(\varepsilon-1)}{\varepsilon}\right]+\frac{2}{5} \ln 2 \approx 0.13 .
\end{gathered}
$$

We note that within the tunnel model, ${ }^{2,3}$ the role of the cut-off factor is played by the quantity $\mathrm{eV}$, which enters large logarithmic factor in the expression for the current. In our case, according to the adiabaticity criterion (12), the effective cut-off factor $\gamma^{4 / 5} \sim \delta E$ is much larger. From this we conclude that within the region of applicability of the adiabatic approach $\mathrm{eV} \ll \delta E$, the quasiparticle and the interference currents are logarithmically smaller as compared to the results of the tunnel model. While the voltage increases and exceeds the depth of the Andreev band $\mathrm{eV}>\delta E$, the tunnel model approximation for the currents $I_{2}$ and $I_{3}$ becomes valid.

Due to the presence of a time-dependent factor $\gamma(t)$ in the logarithmic terms, the currents $I_{2}$ and $I_{3}$ oscillate in time and exhibit logarithmic singularities when $\gamma(t)$ turns to zero, i.e., at $\phi=0$. In the vicinity of these points, the Andreev band vanishes, which violates the condition of adiabaticity (12). Following remarks to Eqs. (63) and (64), we can semiquantitatively describe the whole shape of the oscillations of the quasiparticle and interference currents by adding a small regularization term $\mathrm{eV} / \Delta$ to the denominator of the logarithmic argument in Eq. (64).

By averaging Eq. (63) over time, we obtain the dissipative part of the current $I_{2}(t)$ 


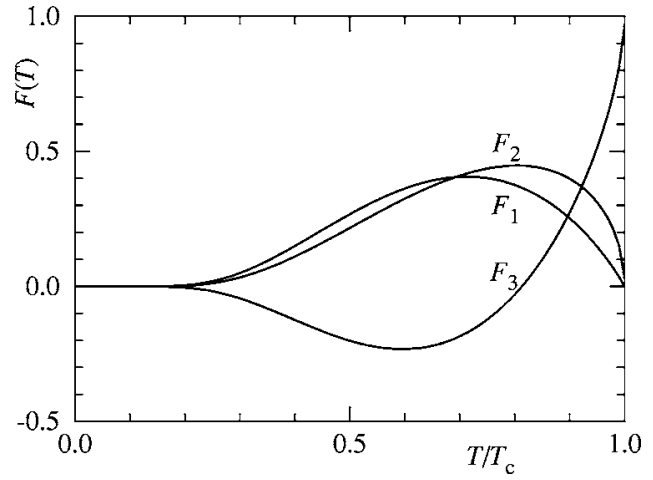

FIG. 6. Temperature dependencies of the functions $F_{1}, F_{2}$, and $F_{3}$.

$$
\begin{gathered}
\left\langle I_{2}\right\rangle_{t}=\frac{V}{R}\left[F_{1} \ln \sqrt{b / W^{4 / 5}}+F_{3}\right], \\
\ln b=\ln a+\int_{0}^{\pi} \frac{d \tau}{\pi} \ln \sin ^{-8 / 5} \tau=1.24, \\
F_{3}(T)=1-n_{\mathrm{eq}}(\Delta)-F_{2}(T) .
\end{gathered}
$$

The interference current also has a dc component due to the oscillations in $I_{3}$, though its magnitude

$$
\begin{gathered}
\left\langle I_{3} \cos \phi\right\rangle_{t}=\frac{V}{R} F_{1} \ln \sqrt{c}, \\
\ln c=\int_{0}^{\pi} \frac{d \tau}{\pi} \cos 2 \tau \ln \sin ^{-8 / 5} \tau=0.8
\end{gathered}
$$

is small with respect to the logarithmic term in $\left\langle I_{2}\right\rangle$. The sum of the quasiparticle and interference dc currents is given by expression

$$
I_{23}^{\mathrm{dc}} \equiv\left\langle I_{2}+I_{3} \cos \phi\right\rangle_{t}=\frac{V}{R}\left[F_{1} \ln \left(2.77 / W^{2 / 5}\right)+F_{3}\right] .
$$

The temperature dependencies of the functions $F_{1}(T), F_{2}(T)$, and $F_{3}(T)$ are shown in Fig. 6.

\section{DISCUSSION}

Analyzing different calculated current components, we conclude that the most significant deviation from the results of the tunnel model comes from the nonequilibrium Josephson current, while the quasiparticle and interference currents undergo minor (logarithmic) changes. There are the two features to be mentioned. The first is a nonmonotonous voltage dependence of the current of trapped quasiparticles described by the relaxation factor $K(\mathrm{eV})$ [see Eqs. (61) and (62)], which has a maximum at $\mathrm{eV} \sim \tau_{\varepsilon}^{-1}$. Due to a rather large value of the parameter $\tau_{\varepsilon} \Delta \gtrsim 10^{2}$ in most superconductors, this current may exceed linear contributions of the quasiparticle and interference currents at moderately small values of the transparency parameter $W$. In this case, the $I-V$ charac-

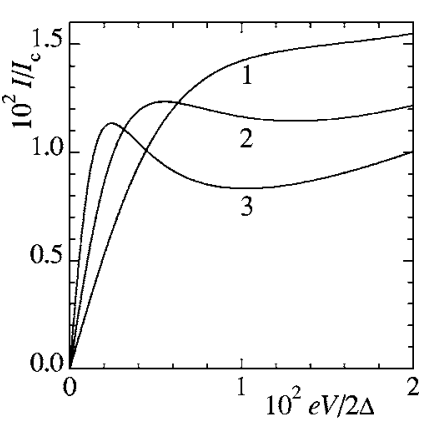

a)

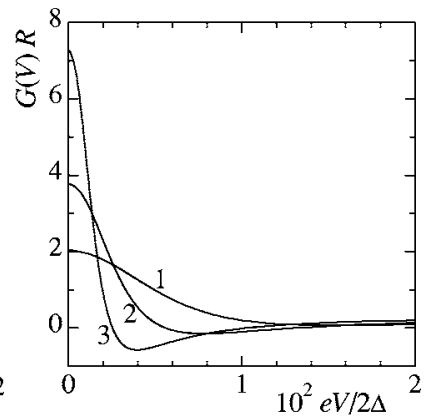

b)
FIG. 7. de current (a) and differential conductance (b) versus voltage for different values of $\tau_{\varepsilon} \Delta=50$ (1), 100 (2), and 200 (3). All curves are plotted at $W=0.05, T=0.5 T_{c}$.

teristic exhibits an $\mathcal{N}$-like feature, as shown in Fig. 7(a), and the linear conductance at a small bias considerably (by the factor $W^{4 / 5} \tau_{\varepsilon} \Delta$ ) exceeds the tunnel model conductance, which is relevant for a larger bias [zero bias conductance peak, Fig. 7(b)].

The second feature is a nonlinear, proportional to $\sqrt{V}$, contribution to the current-voltage characteristics produced by the traveling quasiparticles. We note, however, that because of a small numerical value of the constant $C_{3}$ in Eq. (59), the crossover of $I(V)$ to nonlinear behavior for reasonable values of $W$ actually occurs at very small voltage. The reason for this is a numerically small magnitude of the supercurrent spectral density $I_{s}(E)$ above the bulk gap edge, as is obvious from Fig. 4(a). Physically, this is due to a rapid decrease of the probability of the "over-the-barrier" Andreev reflection (reflection at the energy outside the energy gap). As a result, the density of nonequilibrium quasiparticles produced by the oscillations of $I_{s}$ at $E>\Delta$ appears to be small as compared with the "natural" width $\delta E$ of the perturbed spectral region, which results in smaller contribution of the traveling quasiparticles compared to the trapped ones.

In order to estimate the characteristic parameters of the junction for the transparency parameter $W=0.05$ accepted in our numerical calculations, we will assume the junction area to be $200 \times 200 \mathrm{~nm}$ and the thickness of the leads as well as the elastic scattering length to be $50 \mathrm{~nm}$. For Al leads, this results in the sheet resistance $R_{\square} \approx 0.3 \Omega$ and $R\left(\xi_{0}\right)$ $\approx 0.45 \Omega$ at $\xi_{0} \approx 300 \mathrm{~nm}$. Then, according to Eq. (9), the tunneling probability, the junction resistance, and the critical current approach the values $\Gamma \approx 0.01, R \approx 4.5 \Omega$, and $I_{c}$ $\approx 70 \mu \mathrm{A}$, respectively. Thus, the characteristic voltage region, at which the nonequilibrium dc current dominates, is of the order of several microvolts.

It follows from the presented estimates and also from Fig. 7 that the nonequilibrium effect appears at a rather small applied voltage, which makes it difficult to observe in practice because of the jumps to the Josephson branch. To facilitate the observation, the net Josephson current must be suppressed by applying magnetic field or using the $\mathrm{dc}$ superconducting quantum interference device (SQUID) setup. Remarkably, the effect survives even in the absence of the net Josephson current: Local Josephson currents flowing through different junctions (or parts of the junction) generate 
dissipative dc current flowing in the same direction, which is determined by the applied voltage (i.e., time derivative of the phase) rather than the local values of the phase difference. Indeed, let us consider two junctions connected in parallel (dc SQUID) and apply half-integer magnetic flux. Then the phase differences at the junctions will be shifted by $\pi$. This will lead to different signs of the current spectral density in Eq. (61) for different junctions, however, the nonequilibrium distribution function will also change the sign, which can be proven by direct calculation. Actually, the equality of dc currents in both junctions follows from a simple fact that the constant phase shift is equivalent to the change of the time reference point, which obviously cannot affect the value of the time-averaged (dc) current. Thus, the net dc current will double while the ac current disappears. Such a situation resembles the case of a long SNS junction, ${ }^{10,24}$ where the Josephson effect is suppressed due to the decay of the superconducting correlations inside the normal part of the junction, and the nonequilibrium effect can be observed at a very small applied voltage.

It is instructive to compare the mechanism of nonequilibrium tunnel current in diffusive junctions with a mesoscopic picture of superconductive tunneling in point contacts given by the MAR theory. ${ }^{5,7,8}$ It has been already mentioned in the Introduction, that the Andreev band is the qualitative analog of the Andreev bound levels in point contacts, both are formed by the same physical mechanism-the Andreev reflection by the jump of the superconducting phase at the junction. The time oscillations of the Andreev levels in voltage biased point contacts, and the quasiparticle exchange between the levels and the continuum is the adiabatic description in the time domain of the coherent MAR. ${ }^{8}$ The latter also includes the quasiparticle transitions across the energy gap, which in the real-time picture correspond to the LandauZener tunneling between the Andreev levels (bands). ${ }^{7}$ Such tunneling provides the flow of quasiparticles along the energy axis (spectral flow), proportional to the gradient of the distribution function in the energy space $\partial_{E} f$ within the subgap region $|E|<\Delta$. These processes are weak at a small voltage compared to the continuum-bound level exchange, and they are neglected within the adiabatic approximation adopted in the present paper. For this reason, the spectral flow of the trapped quasiparticles is blocked, which results in a flat (energy-independent) distribution (54) of these quasiparticles in the absence of inelastic relaxation.

However, there is a considerable quantitative difference between the point contacts and diffusive junctions: In the point contacts, most of the quasiparticles reflected by the tunnel barrier escape to the reservoir, while in the long diffusive lead, the quasiparticles multiply and collide with the barrier due to the impurity backscattering. This essentially increases the probability of the coherent tunneling and Andreev reflection ${ }^{25}$ and, correspondingly, enhances the effect of the phase difference on the junction spectrum. As a result, the depth of the Andreev band in our long-arm geometry, $\delta E \sim \Delta W^{4 / 5}$ in Eq. (23), considerably exceeds the depth $\sim \Delta \Gamma$ of the Andreev level in a point contact with comparable transparency.

It is interesting to mention that generally the role of the junction transparency in tunnel junctions with diffusive elec- trodes is played by the parameter $W$ rather than the barrier transparency $\Gamma{ }^{11,12}$ Indeed, comparison of the right- and lefthand sides of Eqs. (8) shows that as long as the "natural" scale of the currents $\hat{J}$ and $\hat{J}^{K}$ is proportional to the gradients of the Green's and distribution functions in the vicinity of the barrier $\sim \xi_{0}^{-1}$, it is the magnitude of $W$ (not $\Gamma$ itself) which determines the effective barrier strength. For example, at large $W \gg 1$, the commutators in Eqs. (8) are to be small, which implies continuity of all functions at the barrier. From this we conclude that at $W \gg 1$, the phase and voltage are continuously distributed along the whole structure, and the barrier does not affect the current transport even if its transparency is small $\Gamma \ll 1$, provided $\Gamma \gg \ell / \xi_{0}$. In other words, at large $W$, the critical current of the tunnel junction, formally estimated by the Ambegaokar-Baratoff formula for a lowtransparent junction, ${ }^{26}$ exceeds the critical current of diffusive superconducting leads. Physically, this effective "blooming" of an opaque barrier results from the multiple coherent backscattering of quasiparticles by impurities mentioned above..$^{25}$

\section{CONCLUSIONS}

We have studied the current transport through the voltage biased tunnel junction with long diffusive superconducting leads at low applied voltage $\mathrm{eV} \ll 2 \Delta$ and finite temperatures. In contrast to the tunnel model, ${ }^{2,3}$ we consider the nonequilibrium effects in the spectrum and distribution of quasiparticles in the vicinity of the barrier. Using the small value of the Josephson frequency with respect to other characteristic energies of the problem, we apply a quasiadiabatic approach to the analysis of the time evolution of quasiparticles. Within such an approach, we obtain a physically transparent picture of the quasiparticle spectrum adiabatically following the time-dependent difference of the superconducting phases. This results in local oscillations of the spectrum of traveling quasiparticles $(E>\Delta)$ and the formation of oscillating Andreev bound states (Andreev band) within the subgap region, $E_{*}(t)<E<\Delta$, at the distance of the order of the coherence length $\xi_{0}$ near the barrier. The quasiparticles trapped by the Andreev band are completely dragged by oscillations of the junction spectrum which reflects complete multiple Andreev reflection (MAR) of the subgap quasiparticles and results in the generation of higher odd harmonics of the ac Josephson current. The inelastic relaxation of the trapped quasiparticles produces a nonadiabatic component of their distribution which manifests itself in the nonequilibrium contribution to the dc current. At low enough voltages, this contribution may considerably exceed the quasiparticle dc current given by the tunnel model; by this reason, the resulting $I-V$ characteristic shows a $\mathcal{N}$-like feature, with the maximum at $\mathrm{eV} \sim \tau_{\varepsilon}^{-1}$. The traveling quasiparticles also deviate from equilibrium due to partial drag (which corresponds to the over-the-barrier MAR) confined by their fast diffusion from the barrier. This results in the additional contribution to the dc current, proportional to $\sqrt{V}$. We note that our approach can be easily extended to the current bias regime; in this case, an arbitrary time-dependent phase must be assumed in the calculation. 
The effect of traveling quasiparticles is interesting, in principle it dominates at a small voltage because of the nonanalytical voltage dependence. Unfortunately, this contribution is numerically small in the case of the tunnel barrier considered here, because of the small spectral density of the Josephson current above the gap. However, there are no fundamental reasons to expect this contribution to be small in other junctions. It would be interesting to find favorable junction geometry.

\section{ACKNOWLEDGMENTS}

We acknowledge useful discussion with L. Kuzmin and D. Winkler. Support from the Swedish grant agencies SSF OXIDE, VR, and KVA is gratefully acknowledged.
${ }^{1}$ I. Giaever, Rev. Mod. Phys. 46, 245 (1974); B. D. Josephson, ibid. 46, 251 (1974).

${ }^{2}$ N. R. Werthamer, Phys. Rev. 147, 255 (1966).

${ }^{3}$ A. I. Larkin and Yu. N. Ovchinnikov, Zh. Eksp. Teor. Fiz. 51, 1535 (1966) [Sov. Phys. JETP 24, 1035 (1967)].

${ }^{4}$ J. R. Schrieffer and J. W. Wilkins, Phys. Rev. Lett. 10, 17 (1963); J. W. Wilkins in Tunneling Phenomena in Solids (Plenum, N.Y., 1963), p. 333.

${ }^{5}$ E. N. Bratus', V. S. Shumeiko, and G. Wendin, Phys. Rev. Lett. 74, 2110 (1995).

${ }^{6}$ A. Furusaki and M. Tsukada, Phys. Rev. B 43, 10164 (1991).

${ }^{7}$ D. Averin and A. Bardas, Phys. Rev. Lett. 75, 1831 (1995); A. Bardas and D. V. Averin, Phys. Rev. B 56, R8518 (1997).

${ }^{8}$ E. N. Bratus', V. S. Shumeiko, E. V. Bezuglyi, and G. Wendin, Phys. Rev. B 55, 12666 (1997).

${ }^{9}$ L. G. Aslamazov and A. I. Larkin, Zh. Eksp. Teor. Fiz. 70, 1340 (1976) [Sov. Phys. JETP 43, 698 (1976)]; A. Schmid, G. Schön, and M. Tinkham, Phys. Rev. B 21, 5076 (1980).

${ }^{10}$ S. V. Lempitskiı̌, Zh. Eksp. Teor. Fiz. 85, 1072 (1983); [Sov. Phys. JETP 58, 624 (1983)].

${ }^{11}$ M. Yu. Kupriyanov, JETP Lett. 56, 399 (1992).

${ }^{12}$ E. V. Bezuglyi, E. N. Bratus', and V. P. Galaiko, Fiz. Nizk. Temp. 25, 230 (1999); [Low Temp. Phys. 25, 167 (1999)].

${ }^{13}$ A. I. Larkin and Yu. N. Ovchinnikov, in Nonequilibrium Superconductivity, edited by D. N. Langenberg and A. I. Larkin (Elsevier, Amsterdam, 1986).
${ }^{14}$ W. Belzig, F. K. Wilhelm, C. Bruder, G. Schön, and A. D. Zaikin, Superlattices Microstruct. 25, 1251 (1999).

${ }^{15}$ M. Yu. Kupriyanov and V. F. Lukichev, Zh. Eksp. Teor. Fiz. 94, 139 (1988) [Sov. Phys. JETP 67, 1163 (1988)].

${ }^{16}$ Yu. N. Ovchinnikov, Zh. Eksp. Teor. Fiz. 59, 128 (1970); [Sov. Phys. JETP 32, 72 (1971)].

${ }^{17}$ S. N. Artemenko, A. F. Volkov, and A. V. Zaitsev, Zh. Eksp. Teor. Fiz. 26, 1816 (1979); [Sov. Phys. JETP 49, 924 (1979)].

${ }^{18}$ A. Brinkman, A. A. Golubov, H. Rogalla, F. K. Wilhelm, and M. Yu. Kupriyanov, Phys. Rev. B 68, 224513 (2003).

${ }^{19}$ K. D. Usadel, Phys. Rev. Lett. 25, 507 (1970).

${ }^{20}$ E. V. Bezuglyi and V. Vinokur, Phys. Rev. Lett. 91, 137002 (2003).

${ }^{21}$ C. J. Lambert and R. Raimondi, J. Phys.: Condens. Matter 10, 901 (1998).

${ }^{22}$ V. P. Galaiko and V. S. Shumeiko, Fiz. Nizk. Temp. 1, 1253 (1975); [Sov. J. Low Temp. Phys. 1, 599 (1975)]; A. Yu. Azovskii, E. V. Bezuglyi, and V. P. Galaiko, Fiz. Nizk. Temp. 10, 818 (1984); [Sov. J. Low Temp. Phys. 10, 428 (1984)].

${ }^{23}$ K. E. Nagaev, Phys. Rev. Lett. 86, 3112 (2001); E. V. Bezuglyi, E. N. Bratus', V. S. Shumeiko, and G. Wendin, Phys. Rev. B 63, 100501(R) (2001).

${ }^{24}$ N. Argaman, Superlattices Microstruct. 25, 861 (1999).

${ }^{25}$ B. J. van Wees, P. de Vries, P. Magneè, and T. M. Klapwijk, Phys. Rev. Lett. 69, 510 (1992).

${ }^{26}$ V. Ambegaokar and A. Baratoff, Phys. Rev. Lett. 10, 486 (1963). 